\title{
La valeur ontologique de l'image et de la ressemblance de l'être humain avec Dieu en bioéthique
} The Ontological Value of the Image and Resemblance of the human being to God in
Bioethics

Francisco Quesada Rodriguez

Universidad de Costa Rica, Costa Rica

\section{Résumé}

Cet article expose quelques réflexions théologiques et éthiques inspirées de la conférence «Comment pouvons-nous justifier notre devoir envers les générations futures et la terre indépendamment de la foi ? » prononcée par Hans Jonas à Munich en 1984 à l'occasion de la $88^{\mathrm{e}}$ Journée catholique allemande. D'après Jonas, il ne s'agit pas d'exclure la religion de l'éthique, mais de bien donner une place à la religion et à la théologie dans l'ontologie pour bien fournir le débat sur l'éthique environnementale et la bioéthique. La question de la valeur ontologique de l'image et la ressemblance de l'être humain avec Dieu peut se comprendre en rapport à la «Preimplantation Genetic Diagnosis 》 (PGD) et la «Preimplatantion Genetic Screening » (PGS). L'examen des livres Das Prinzip Verantwortung (1979) et Technik, Medizin und Ethik (1985) permet de comprendre le rôle de la religion, du gnosticisme et de la théologie en ce qui concerne la bioéthique de la responsabilité selon Hans Jonas.

\footnotetext{
Abstract

This article presents some theological and ethical reflections inspired by the conference "How can we justify our duty to future generations and to the earth regardless of faith?" delivered by Hans Jonas in Munich in 1984 on the occasion of the $88^{\text {th }}$ German Catholic Day. According to Jonas, it is not a question of excluding religion from ethics, but of giving a place to religion and theology in ontology to properly provide the debate on environmental ethics and bioethics. The question of the ontological value of the image and resemblance of the human being to God can be understood in relation to "Preimplantation Genetic Diagnosis" (PGD) and "Preimplatantion Genetic Screening" (PGS). An analysis of the books Das Prinzip Verantwortung (1979) and Technik, Medizin und Ethik (1985) sheds light on the role of religion, Gnosticism and theology in the bioethics of responsibility according to Hans Jonas.
}

Palavras-chave

Hans Jonas. Théologie. Création. Gnosticisme. Responsabilité.

\section{Keywords}

Hans Jonas. Theology. Creation. Gnosticism. Responsibility. 


\section{Introduction}

Dans la conférence «Comment pouvons-nous justifier notre devoir envers les générations futures et la terre indépendamment de la foi ? »1, Hans Jonas s'adresse aux théologiens catholiques pour donner un avis philosophique sur la fondation ontologique d'une obligation pour la postérité de l'humanité et de la terre sans dépendance religieuse. Certes, ce que Jonas a voulu démontrer dans l'impératif catégorique-existentiel est que la responsabilité humaine doit être ancrée dans l'ontologie de l'être vivant, sans penser pour autant que l'argument philosophique est incompatible avec la foi, la religion et la théologie. Au contraire, la théologie peut nourrir la discussion sur l'être humain et la nature dans le cadre de la civilisation technologique. En fait, selon le récit biblique du livre de la Genèse, la responsabilité envers Dieu, la création et l'être humain montre qu'il y a une valeur fondamentale de la vie. Loin d'exclure la foi et la religion, la théologie biblique peut questionner la philosophie sur la bonté de la création et de l'être humain au sens ontologique, mais la théologie doit s'exprimer aussi en langage rationnel et l'herméneutique de démythologisation peut être bien comprise par la philosophie.

L'être humain peut disparaître s'il n'agit pas de façon responsable envers lui-même avec l'usage de la technologie. " 'L'image et la ressemblance" » de l'être humain avec Dieu montre qu'il y a une valeur fondamentale dans l'humanité, laquelle demande une explication théologique, face au dualisme gnostique qui méprise la vie dans le monde ainsi que la nature et le corps humain en tant que matière. Même si l'éthique ne peut pas se fonder sur la théologie, la théologie peut poser des questions métaphysiques radicales selon l'ordre de la création («ordo creationis ») pour ne pas tomber dans le nihilisme.

\footnotetext{
1 JONAS, Hans. Wie können wir unsere Pflicht gegen die Nachwelt und die Erde unabhängig vom Glauben begründen?. In: BÖHLER, Dietrich; BRUNE, Jens Peter. Orientierung und Verantwortung. Begegnungen und Auseinandersetzungen mit Hans Jonas. Würzburg: Königshausen \& Neumann, 2004, p. 71-84 (HJ 8-13-22). (88. Deutsche Katholikentag in München: 4.-8. 7. 1984. Titel: „Dem Leben trauen, weil Gott es mit uns lebt“). 
535 | La valeur ontologique de l'image et de la ressemblance de l'être humain avec Dieu...

La philosophie doit se questionner sur la valeur ontologique de l'être humain sur terre face à l'intervention technologique sur la nature humaine. Les techniques de diagnose génétique prénatale et/ou préimplantatoire telles que «Preimplantation Genetic Diagnosis » (PGD) et «Preimplatantion Genetic Screening » $(P G S)$ permettent de prévenir des maladies génétiques, certes, mais au risque de changer la nature humaine par la manipulation génétique et de dévier vers une sorte d'eugénisme social. C'est ainsi que la préservation de l'image de l'être humain et la paternité en tant qu'archétype de la responsabilité sont les deux critères de la bio-éthique de la responsabilité conçue par Hans Jonas, qui fonctionne en ce qui concerne le défi de la technoscience pour l'humanité.

\section{La responsabilité de l'être humain envers la création de Dieu}

La technologie moderne a changé la manière dont l'être humain habite le monde : " un nouveau facteur est intervenu dans l'équation humaine et planétaire. Son nom est "la technologie moderne" $»^{2}$. C'est un «pouvoir humain » qui dépasse l'agir spatiotemporel de l'être humain lui-même et met à mal également le «monopole anthropocentrique» de la plupart des systèmes éthiques précédents, soit religieux soit séculiers. Le pouvoir technologique dépasse aussi la capacité de «l'amour du prochain » (cf. Matt 22,39 ) et touche la vie dans la biosphère de la planète, la nature ou la création de Dieu. Il y a donc un excès de pouvoir par rapport au rôle que la religion judéo-chrétienne avait attribué à l'être humain, d'être l'administrateur ou le tuteur de la création selon le livre de la Genèse (cf. Gn $1,28)$. D'après Jonas, il est possible de se questionner à partir du récit biblique de la création au niveau philosophique :

Et en fait, le récit biblique de la création contient pour le croyant - en particulier l'affirmation que Dieu a bien fait son œuvre, qu'il a créé l'homme à son image et lui a donné la domination sur toutes les autres créatures - la complète

2 JONAS, 2004, p. 72. 
justification recherchée de la domination par son devoir concomitant de préserver l'intégrité de la création divine, en lui-même et dans son milieu de vie. Oui, même la célèbre - si facilement résolue apparemment - première question de la métaphysique, pourquoi tout est au lieu de rien, trouve la réponse dans le jugement divin, ce qui est créé est bon, donc son être est mieux que rien. Ici, le croyant doit être envié. Mais je veux montrer brièvement pourquoi il est nécessaire, par conséquent, d'établir le dit devoir indépendamment de la foi par la raison, pour le fonder philosophiquement ....

Évidemment, Jonas fait allusion ici au livre de la Genèse (cf. Gn 1, 2628). Certes, la croyance en Dieu pour Jonas a une place privilégiée parce que tout a été créé «bon » par Dieu, mais il faut aller plus loin pour chercher une justification ontologique de la bonté de la création et de l'être humain, sans nier la richesse du récit biblique. Par son interprétation philosophique, Jonas ne détache pas l'être humain de la nature, mais il essaie de justifier une ontologie de la bonté de l'image de l'être humain dans la nature à partir de la demande fondamentale de la métaphysique exprimée par Leibniz, à savoir : «Pourquoi y a-t-il quelque chose plutôt que rien ? » ${ }^{4}$. Jonas donne deux raisons pour lesquelles il est nécessaire de fournir une justification philosophique indépendante de la foi :

Une justification indépendamment de la foi est désormais nécessaire parce que, c'est reconnu, beaucoup ne croient plus aujourd'hui au Dieu judéo-chrétien et à la création du monde par lui, donc à tout le récit du premier chapitre de la Genèse. En outre, une raison peut être donnée pour l'urgent devoir commun de la responsabilité humaine (une "Summa contra gentiles », pour ainsi dire). Mais la réalité elle-même ne détache pas la foi de la question de la raison et le croyant aussi doit s'y soumettre ${ }^{5}$.

Premièrement, le problème de l'incrédulité en Dieu et à la création divine selon le récit du livre de la Genèse impose aussi une justification rationnelle pour soutenir la bonté de la création. Et Jonas fait référence au livre quatre de la Summa contra gentiles de Saint Thomas d'Aquin, dans

\footnotetext{
3 JONAS, 2004, p. 74.

${ }^{4}$ LEIBNIZ, Gottfried Wilhelm. Principes de la nature et de la grâce fondés en raison. Principes de la philosophie ou Monadologie. Paris: PUF, 1954, p. 45 [§7].

5 JONAS, 2004, p. 74.

Fronteiras, Recife, v. 4, n. 2, p. 533-553, jul./dez., 2021
} 
537 | La valeur ontologique de l'image et de la ressemblance de l'être humain avec Dieu...

lequel il y a une défense du mystère chrétien à partir de la raison. Deuxièmement, le croyant doit s'engager par une justification rationnelle pour ne pas détacher la foi de la réalité, comme a fait le théologien dominicain.

Cependant, la question «pourquoi » Dieu a créé le monde «bon» (voir par exemple le livre de la Genèse ainsi que le Timée de Platon) ne peut pas trouver réponse tout simplement par une explication du «pouvoir » de cause et d'effet, mais doit partir de la bonté de Dieu lui-même et du jugement divin, et pas uniquement de la volonté divine, comme a essayé de l'exprimer le bienheureux Jean Duns Scot. Il faut aller au-delà de la simple compréhension du jugement divin par la seule piété, il est nécessaire de trouver une explication rationnelle à partir de la formule Fides quaerens intellectum de Saint Anselme de Canterbury.

Afin d'exprimer le pourquoi de la «bonne » création, Jonas reprend la question de Leibniz. Le philosophe Leibniz a postulé que Dieu avait dans la création du monde ses propres raisons, bien sûr raisonnables, et que notre raison peut les penser et les découvrir. Cela signifie que la question du devoirêtre du monde peut être séparée de toute thèse sur son auteur et de la doctrine de la création :

En d'autres termes : la question du devoir-être d'un monde est séparable de toute thèse concernant son auteur, précisément en supposant que même pour un créateur divin un tel devoir-être conforme au concept de Bien était la raison de son acte créateur: il voulait parce qu'il estimait qu'il devait être. On peut même affirmer que la perception d'une valeur dans le monde est une des motivations pour conclure à un auteur divin (autrefois c'était même une des "preuves" de l'existence de Dieu) et non pas qu'au contraire la postulation de l'auteur était la raison d'accorder de la valeur de sa création ${ }^{6}$.

La valeur de la vie est un bien ontologique intrinsèque à l'être vivant et peut-être le vivant même peut-il donner des « vestiges » du Créateur, pour le dire dans la terminologie du théologien franciscain Saint Bonaventure.

6 JONAS, Hans. Le principe responsabilité. Une éthique pour la civilisation technologique. Paris : Flammarion, 2009, p. 103 ; JONAS, Hans. Das Prinzip Verantwortung. Versuch einer Ethik für die technologische Zivilisation. Suhrkamp: Frankfurt am Main, 2003, p. 99. 
Même si on ne peut pas déduire la valeur ontologique de l'être vivant à partir de l'«auteur divin», il ne faut pas exclure cependant la théologie de la métaphysique en raison de «l'éclipse de la foi ». Au contraire, «la métaphysique peut apprendre de la théologie la radicalité de l'interrogation pourquoi il y a quelque chose plutôt que rien, auparavant insoluble dans la philosophique antique ${ }^{7}$. On trouve aussi le même argument tiré du livre Le principe responsabilité dans la conférence donnée en 1984 à Munich ${ }^{8}$.

C'est ainsi que la théologie a une place fondamentale pour la métaphysique. Mais pour bien élever la richesse du récit biblique de la création au niveau philosophique, il faut comprendre le texte par l'herméneutique de la démythologisation, à savoir : une « désobjectivation » ou subjectivation, une «traduction » ou « retraduction » et une « dialectique de concepts ${ }^{9}$. En fait, par l'herméneutique de la démythologisation, on peut dégager et mettre au jour le concept de «bon» de la création, particulièrement face aux défis posés par la technologie à l'être humain et à la nature.

La justification philosophique du pourquoi l'être vivant est « bon » et a une valeur ontologique, Jonas l'a donnée amplement, dans son livre Le principe responsabilité, comme une «objectivation» éthique de la «subjectivité » de l'être humain et de la nature. Certes, dans la fondation ontologique de l'éthique, Jonas n'exclut absolument pas Dieu, mais le devoir doit être fondé dans un bien intrinsèque: «Nous disons donc que le “commandement" ne peut pas émaner seulement d'une volonté qui commande, par exemple d'un Dieu personnel, mais également de la revivification immanente d'un bien en soi qui revendique sa propre effectivité $\gg 10$. Autrement dit, la croyance en Dieu peut motiver l'éthique à partir de la subjectivité, mais il reste nécessaire d'établir l'objectivité du « bien en soi » de la création divine.

\footnotetext{
7 JONAS, 2009, p. 103 ; JONAS, 2003, p. 99.

8 JONAS, 2004, p. 75.

9 JONAS, Hans. Heidegger et la théologie. In: JONAS, Hans. Le phénomène de la vie. Vers une biologie philosophique. Bruxelles: De Boeck, 2001, p. 261; JONAS, Hans. Heidegger and the Theology. In: JONAS, Hans. The Phenomenon of Life. Toward a Philosophical Biology. Illinois: Northwestern University Press, 1996, p. 260.

10 JONAS, 2009, p. 157; JONAS, 2003, p. 153.
}

Fronteiras, Recife, v. 4, n. 2, p. 533-553, jul./dez., 2021 
539 | La valeur ontologique de l'image et de la ressemblance de l'être humain avec Dieu...

En effet, pour Jonas il y a un «devoir biblique » de préserver " "l'image et la ressemblance" » de l'être humain avec Dieu selon le livre de la Genèse, qui ne peut pas disparaître face aux désirs d'amélioration technoscientifique ${ }^{11}$. Il s'agit d'un devoir ontologique de préserver " "l'image et la ressemblance" » $(G n 1,27)$ de l'être humain face aux chances et risques que présentent la science et la technologie. Dans le livre Le principe responsabilité, Jonas a affirmé le sens «sacré » de l'être humain, malgré l'exclusion de la religion du domaine technoscientifique :

Préserver l'héritage dans son intention qui vise "l'image et la ressemblance", donc négativement le protéger également contre la dégradation, c'est là l'affaire de tout instant; ne pas tolérer de pauses en cela est la meilleure garantie de la durée; c'est, sinon la garantie, du moins la condition préalable même de l'intégrité future de “l'image et la ressemblance" 12 .

Il ne s'agit pas de préserver tout simplement l'essence de l'humanité au sens métaphysique traditionnel, mais de garantir l'existence humaine au sens ontologique pour les futures générations, comme Jonas l'a proposé dans l'impératif catégorique-existentiel, lequel demande que l'agir de l'être humain soit compatible avec la vie humaine sur terre :

"Agis de façon que les effets de ton action soient compatibles
avec la permanence d'une vie authentiquement humaine sur
terre"; ou pour l'exprimer négativement : “Agis de façon que
les effets de ton action ne soient pas destructeurs pour la
possibilité future d'une telle vie"; simplement : “Ne
compromets pas les conditions de la survie indéfinie de
l'humanité sur terre"; ou encore, formulé de nouveau
positivement: "Inclus dans ton choix actuel l'intégrité future
de l'homme comme objet secondaire de ton vouloir"'.

Dans l'impératif catégorique-existentiel, Jonas demande une vie humaine authentique, que soit possible une vie humaine, la survie de l'humanité sur terre et l'intégrité de l'être humain. Il ne s'agit pas d'un impératif anthropocentrique car il considère aussi la vie sur notre terre ; la

\footnotetext{
11 JONAS, 2004, p. 85.

12 JONAS, 2009, p. 424 ; JONAS, 2003, p. 393.

13 JONAS, 2009, p. 40 ; JONAS, 2003, p. 36.
} 
vie humaine n'est pas possible sans rapport à la nature. Enfin, selon la conclusion de Das Prinzip Verantwortung, il faut préserver « "l'image et la ressemblance" » comme une responsabilité de l'être humain envers lui-même dans l'avenir.

Le théologien Karl Lehmann (1936-2018) a remarqué aussi la « responsabilité » de l'être humain envers la création à partir de l'interprétation de « “l'image et la ressemblance” » que fait Jonas du livre de la Genèse. Même si le récit biblique de la création n'est évidemment pas historique, il a une fonction importante pour l'évaluation de la technique moderne ${ }^{14}$. Il est donc possible de dire que ce que le cardinal Lehmann a esquissé de l'anthropologie jonassienne est dans quelque mesure ce que Habermas avait signalé auparavant : le «premier ouvrage [de Hans Jonas] sur le gnosticisme a été reconnu dans la discussion théologique, cependant ses œuvres philosophico-anthropologiques attendent encore une réception productive élargie $\gg 15$.

\section{Théologie et gnosticisme dans Le principe responsabilité}

Bien que Jonas n'exclue pas totalement Dieu pour bien justifier la bioéthique de la responsabilité, il souligne que la religion et la théologie doivent protéger l'éthique de l'influence du «dualisme » et de l'ontologie «"gnostique" » impliquant une instance du dehors qui désenchante la nature :

\footnotetext{
Mais avant tout je peux seulement avoir légitimement le droit d'entrer en dissentiment avec la nature, si je peux invoquer une instance en dehors d'elle, c'est-à-dire, une transcendance qui à son tour possède l'autorité qui est refusée à celle-là : donc sous la condition de quelque dualisme. (Car le dissentiment du simple goût serait frivole). Ce dualisme devrait travailler avec la théologie d'un dieu qui, soit ne
}

14 LEHMANN, Karl. Auslotungen. Lebensgestaltung aus dem Glauben heute. Freiburg im Breisgau: Herder, 2016, p. 194, 195, 203. Lehmann a cité ici la Kritische Gesamtausgabe der Werke von Hans Jonas (I/2, Tbd. 1).

${ }^{15}$ HABERMAS, Jürgen. Im Sog der Technokratie. Kleine Politische Schriften XII. Frankfurt am Main: Suhrkamp, 2013, p. 18-19. 
541 | La valeur ontologique de l'image et de la ressemblance de l'être humain avec Dieu...

serait nullement responsable pour le monde, soit qui serait contrecarré par un principe contraire lors de la création de celui-ci, soit qui le créerait lui-même de travers, dans un dessein supérieur. De plus il devrait travailler avec la théologie d'un monde corrélativement mauvais (et pas seulement indifférent). En outre, il devrait travailler dans tous les cas avec une théorie de l'âme transcendante qui accomplit cet acte de dissentiment : une théorie "gnostique" de l'être, qui est sans doute la dernière chose qu'accepterait n'importe quel protagoniste de ce débat. Sous des conditions monistes par contre, ne serait possible qu'un dissentiment légitime au niveau du particulier mais non pas au niveau de la totalité ${ }^{16}$.

D’après Jonas, on peut tirer cinq conceptions théologiques dérivées du dualisme pour être en désaccord avec la nature. Premièrement, une sorte de dieu démiurge qui n'est pas responsable du monde ; deuxièmement, un dieu contrarié par une force opposée dans la création telle que le mal ; troisièmement, un dieu qui a engendré par un processus, à partir d'un dessein supérieur; quatrièmement, un monde mauvais avec lequel la théologie devrait travailler ; cinquièmement, en ce qui concerne l'anthropologie, le dualisme affecte la dimension somatique à partir de l'idée d'une « âme transcendante ». C'est ainsi que la théologie a la tâche de protéger la nature contre toute forme de dualisme et de gnosticisme qui désenchante la nature. La théologie de la création peut prévenir l'éthique contre le mépris de la nature.

À cet égard, une éthique théologique ou une éthique religieuse de «l'accomplissement au-delà », qui attend le salut de l'âme dans la transcendance, jouit de l'existence comme une vie agréable à Dieu dans le présent, mais elle peut se détacher du monde, comme le gnosticisme, par un renoncement dans lequel la vie perd de la valeur en soi dans le présent et dans le futur :

Si donc l'on demande pourquoi le renoncement radical ici-bas est considéré comme suffisamment méritoire pour pouvoir s'attendre à ce dédommagement ou à cette récompense, une réponse peut être que la chair est pécheresse, que le plaisir est mauvais et que le monde est impur et dans ce cas (comme dans celui, légèrement différent, où l'individuation comme

16 JONAS, 2009, p. 153-154; JONAS, 2003, p. 148-149. 
telle est censée être mauvaise), l'ascèse comporte malgré tout de nouveau une instrumentalité authentique de l'agir et un chemin de la réalisation interne des fins par l'agir personnel - à savoir le chemin qui mène de l'impureté à la pureté, de l'état du pécheur à la sainteté, de l'état servile à la liberté, de l'égoïsme au renoncement à soi : pour autant qu'elle est tout cela, sous de telles conditions métaphysiques, l'ascèse elle-même est donc déjà la meilleure des manières de vivre. Mais de cette façon nous serions de nouveau revenus à l'éthique de l'immédiateté et de la simultanéité - une forme sans doute hautement égoïste et extrêmement individualiste de l'éthique de la perfection de soi, à laquelle il est également donné, dans les mouvements d'illumination spirituelle auxquels ses efforts peuvent aboutir, de jouir déjà de la récompense éternelle dans l'expérience mystique de l'absolu.

In summa, pouvons-nous dire, pour autant que ce complexe de l'orientation vers un but transcendant relève de l'éthique - ce qu'il fait en particulier dans la forme "modérée" d'une vie agréable à Dieu comme condition de la récompense éternelle, mentionnée en premier lieu - il conforme lui aussi notre thèse selon laquelle toutes les éthiques précédentes ne se déploient que dans le présent ${ }^{17}$.

D’après Jonas, une éthique théologique ou une éthique religieuse de «l'accomplissement au-delà » méprise le corps humain pour trois raisons particulièrement : parce que « la chair est considérée comme pécheresse », « le plaisir comme mauvais » et « le monde comme impur ». L'ascèse est une pratique personnelle qui permet de purifier le corps du monde et de faire ainsi le chemin de la sainteté, mais c'est une pratique égoïste et individualiste qui mène au « renoncement à soi » en tant qu'une « éthique de perfection de soi ». Bref, ce n'est qu'une description de la morale gnostique $^{18}$, qui peut influencer la religion et la théologie, contrairement à la valeur ontologique de la vie. Enfin, l'aspiration théologique et religieuse à la transcendance ne devrait pas se passer de la responsabilité envers le monde, la nature et l'être humain.

\footnotetext{
17 JONAS, 2009, p. 43-45 ; JONAS, 2003, p. 40-42.

18 JONAS, Hans. La Religion Gnostique. Le message du Dieu étranger et les débuts du christianisme. Paris: Flammarion, 1978, p. 61; JONAS, Hans. The Gnostic Religion. The Message of the Alien God and the Beginnings of Christianity. Boston : Beacon Press, 2001, p. 46-47.
} 
543 | La valeur ontologique de l'image et de la ressemblance de l'être humain avec Dieu...

\section{Peut-on donc fonder l'éthique sur la religion et la théologie?}

Dans les livres Das Prinzip Verantwortung (1979) et Technik, Medizin und Ethik (1985), on ne trouve aucune trace d'une fondation théologique de la bioéthique de la responsabilité. Au contraire, Hans Jonas a souligné auparavant dans le livre The Phenomenon of Life (1966) et il a insisté aussi dans Das Prinzip Verantwortung, qu'aucune éthique ne peut être fondée uniquement sur l'autorité divine, la religion ou la théologie. Dans l'épilogue «Nature et éthique» de The Phenomenon of Life, Jonas affirme que: «Une éthique qui n'est plus fondée sur l'autorité divine doit être fondée sur un principe qu'on puisse découvrir dans la nature des choses ".

En tant que telle, l'éthique ne peut être fondée tout simplement sur «l'autorité divine», mais bien doit s'ériger sur l'ontologie et, particulièrement, l'être vivant. D'après Jonas, l'éthique doit se fonder « dans la théorie générale de l'être et de la vie ». Cependant, Jonas n'exclut pas totalement la théologie dans le cadre de la théorie de l'être. L'éthique doit se fonder « dans une assignation objective par la nature des choses (ce que la théologie a l'habitude d'appeler l'ordo creationis ${ }^{19}$. En effet, c'est la théologie qui a découvert la valeur de l'être vivant en tant que création de Dieu, malgré sa perspective théocentrique et anthropocentrique.

En ce qui concerne la fondation ontologique de l'éthique, dans le livre Das Prinzip Verantwortung, Jonas considère que, en cas de absence d'une religion, l'éthique a encore une tâche pour ordonner l'agir de l'être humain : "Mais une religion absente ne saurait décharger l'éthique de sa tâche; et alors qu'on peut dire de la première qu'elle existe ou qu'elle n'existe pas comme fait exerçant une influence déterminante sur les hommes, il faut dire de l'éthique qu'elle doit exister ${ }^{20}$. Même si la religion n'existe pas, l'éthique a encore une tâche fondamentale pour orienter l'agir humain dans le monde avec la métaphysique :

19 JONAS, Hans. Le phénomène de la vie. Vers une biologie philosophique. Bruxelles: De Boeck, 2001, p. 282; JONAS, Hans. The Phenomenon of Life. Toward a Philosophical Biology. Illinois : Northwestern University Press, 1996, p. 284.

20 JONAS, 2009, p. 61; JONAS, 2003, p. 57-58.

Fronteiras, Recife, v. 4, n. 2, p. 533-553, jul./dez., 2021 
Sans doute une métaphysique valable ne peut-elle être fournie, pas plus que la religion, par le simple diktat de l'amère nécessité qui la réclame ; en revanche la nécessité peut nous imposer de la chercher et le philosophe séculier qui s'efforce d'établir une éthique doit au préalable admettre la possibilité d'une métaphysique rationnelle, nonobstant Kant ${ }^{21}$.

La métaphysique peut fournir l'éthique à partir de «l'amère nécessité », pas comme la religion et la foi qui dépendent de l'ordre de la création («ordo creationis»), selon la conception théologique de la Cité de Dieu de Saint Augustin. En fait, la métaphysique peut apprendre de la théologie la radicalité de questionnement des choses créées par Dieu ${ }^{22}$.

La bioéthique de la responsabilité et, particulièrement, l'impératif catégorique-existentiel jonassien peuvent être interprétés en tant que tels, dans le cadre de la philosophie de Hans Jonas, comme une réponse humaine à Dieu par une sorte de mystique, étant donné l'impuissance divine dans le monde et la défiguration de l'image de l'être humain ${ }^{23}$. Ce n'est pas l'impuissance de Dieu qui forge la bioéthique de la responsabilité, mais bien, c'est la bioéthique de la responsabilité qui donne sens à la théologie spéculative comme une interprétation démythologisante de Dieu.

Il est possible de trouver la signifiance du concept «valeur » dans le livre Das Prinzip Verantwortung. D’après Jonas, les valeurs sont des idées forgées par l'être humain, dans le monde, par rapport à l'être, grâce à la capacité eidétique. D’abord, les valeurs sont forgées à partir de l'expérience subjective à l'intérieur de l'être humain, elles sont ensuite exprimées par la pensée et l'agir humain, objectivement, dans le monde ${ }^{24}$.

L'être humain a la capacité eidétique de percevoir et de découvrir, à partir de l'existence de l'être vivant dans la nature et à partir de la réflexion sur soi-même en tant qu'être humain dans le monde, la valeur intrinsèque de la nature. Il s'agit d'une fondation ontologique de la valeur de l'être vivant,

\footnotetext{
21 JONAS, 2009, p. 99; JONAS, 2003, p. 94.

22 JONAS, 2004, p. 75.

23 JONAS, Hans. The Concept of God after Auschwitz. In: JONAS, Hans. Mortality and Morality. A Search for the Good after Auschwitz. Illinois, Evanston: Northwest University Press, 1996, p. 131-143.

${ }^{24}$ RATH, Matthias. La triple signification du mot "valeur" dans Das Prinzip Verantwortung de Hans Jonas et la Psychologisation en éthique. In: HOTTOIS, Gilbert ; PINSART, MarieGeneviève (Éds.). Hans Jonas. Nature et responsabilité. Paris : Vrin, 1993, p. 132-135. 
et particulièrement de l'être humain lui-même parce qu'il appartient aussi à la nature.

Quoi qu'il en soit, créé ou non par Dieu, abstraction faite d'un « créateur divin », l'être vivant a une valeur ontologique intrinsèque qui peut être découverte par l'être humain grâce à la capacité eidétique. C'est grâce à la capacité eidétique que l'être humain peut reconnaitre, non seulement la valeur intrinsèque, mais aussi le devoir-être envers l'être vivant qui le fait responsable de la nature, indépendamment de l'idée d'un créateur divin :

On peut même affirmer que la perception d'une valeur dans le monde est une des motivations pour conclure à un auteur divin (autrefois c'était même une des "preuves" de l'existence de Dieu) et non pas qu'au contraire la postulation de l'auteur était la raison d'accorder de la valeur à sa création $^{25}$.

Certes, pour le croyant la découverte de la valeur de la vie est une raison fondamentale pour réaffirmer l'existence de Dieu. Mais l'existence de Dieu en tant que telle n'est pas la seule raison pour laquelle l'être humain doit soutenir la valeur intrinsèque de l'être humain. C'est ainsi qu'une éthique créée sur des critères religieux ne propose qu'une perspective insuffisante de la vie, parce qu'elle dépend surtout d'une interprétation subjective :

On avait déjà laissé entendre que la foi religieuse dispose ici déjà des réponses que la philosophie ne peut que chercher avec une chance incertaine de succès. (Par exemple, on peut déduire de "l'ordre de la créé" que la volonté de Dieu veut qu'existent des hommes à son image et qu'existe l'ordre entier dans son invulnérabilité). La foi peut donc très bien procurer à l'éthique le fondement, mais elle-même n'est pas disponible sur commande et même en y mettant l'argument le plus fort de l'obligation, on ne peut pas faire appel à celle qui est absente ou discréditée ${ }^{26}$.

Pour le croyant, l'existence de Dieu peut donner un fondement à l'éthique. Mais l'existence de Dieu n'est pas la seule raison pour soutenir le devoir humain envers la nature. En tout cas, selon Jonas, l'être humain a un

25 JONAS, 2009, p. 102-103; JONAS, 2003, p. 98-99.

26 JONAS, 2009, p. 98-99; JONAS, 2003, p. 94-95. 
sens sacré : «die Sakrosanktheit des Subjektes der Entwicklung »27. Or cette expression et d'autres allusions n'évoquent la valeur absolue de la vie que dans un sens séculier, «à la différence du judaïsme et du christianisme » 28 . Même abstraction faite de l'existence de Dieu, on doit reconnaître la sainteté de «l'intégrité de l'image humaine ${ }^{29}$.

\section{Le devoir ontologique envers l'image de l'être humain en bioéthique}

On peut donc se demander comment fonctionne la catégorie ontologique de l'image de l'être humain en bioéthique. Un exemple d'actualité peut nous aider à bien exprimer la bioéthique de la responsabilité : «Preimplantation Genetic Diagnosis» (PGD) et «Preimplatantion Genetic Screening» (PGS). Ce sont des techniques de diagnostic génétique prénatal, développées à partir de l'amniocentèse dans les années 1970. Or depuis les années 1990 jusqu'à nos jours, grâce à la science et à la technologie, c'est-à-dire la technoscience, la PGD et la PGS ont permis de détecter plusieurs anormalités et maladies génétiques avant l'implantation de l'embryon dans l'utérus, posant par là une série de dilemmes bioéthiques pour les généticiens, les médecins, les couples, les parents et la société.

La PGD est une technique appliquée pour détecter les anormalités génétiques dans un seul gène (« single gene ») de couples qui ont quelque risque de transmettre une maladie héréditaire à la progéniture, particulièrement s'ils ont déjà un enfant malade. Pour pratiquer la PGD, le couple doit se soumettre à la Fertilisation in Vitro (IVF) après avoir obtenu un gène spécifique, puis diagnostiquer l'embryon et l'implanter dans l'utérus. La PGS est utilisée pour faire le test génétique des embryons en appliquant les techniques cytogénétiques pour détecter des anormalités chromosomiques

27 JONAS, 2009, p. 76-78; JONAS, 2003, p. 72-75; JONAS, Hans. Technik, Medizin und Ethik. Zur Praxis des Prinzips Verantwortung. Frankfurt am Main: Suhrkamp, 1985, p. 235.

${ }_{28}$ POLIWODA, Sebastian. Versorgung von Sein. Die philosophischen Grundlagen der Bioethik bei Hans Jonas. Zürich/New York: Georg Olms Verlag, 2005, p. 225-229.

29 JONAS, Hans. Ethics and Biogenetic Art . Social Research, Volume 71, Number 3, Fall 2004, p. 581. 
547 | La valeur ontologique de l'image et de la ressemblance de l'être humain avec Dieu...

chez les patients qui pratiquent l'IVF, particulièrement les patients d'âge avancé qui sont infertiles.

Dans ce sens, PGD et PGS sont des techniques différentes, même si elles essaient de prévenir des maladies dans la progéniture, maladies génétiques héréditaires ou anormalités génétiques. Tandis que la PGD essaie de détecter une maladie génétique héréditaire dans la progéniture, la PGS essaie d'améliorer intentionnellement la constitution génétique de l'embryon pour prévenir les anormalités génétiques. Il reste que PGD et PGS sont deux techniques invasives qui posent des dilemmes bioéthiques.

Depuis les années 1990, les techniques de diagnose génétique prénatale se sont ainsi constamment développées grâce à la science et à la technologie, la technoscience. La découverte constante de maladies et d'anormalités génétiques modifie cependant le concept traditionnel de santé et de maladie et pose un dilemme pour la société parce qu'elle peut faire surgir l'idée d'un être humain parfait, nettoyé de maladies et d'anormalités génétiques. La PGD et la PGS mettent en avant l'amélioration de l'être humain et peuvent ainsi devenir une sorte d'eugénisme pour quelques membres de la société. En fait, les parents qui se soumettent à ces techniques peuvent devenir «designer babies» à partir de l'idée d'un «perfect baby ».

C'est pourquoi la responsabilité pour les conséquences de l'agir humain liées au pouvoir technoscientifique doit fonctionner ici comme le critère bio-éthique fondamental pour les généticiens, les médecins, les couples, les parents et la société. La responsabilité peut être comprise selon «l'heuristique de la peur » comme un pronostic des risques ${ }^{30}$.

Afin de donner une orientation bioéthique, il faut dire d'abord que ces techniques offrent des chances ou avantages dans la mesure où elles évitent la douleur et la souffrance pour les enfants, les parents et la société, mais en même temps elles comportent une série de risques ou inconvénients tels que procréation sans sexualité, sélection du sexe de l'enfant, risques de

30 SCHOCKENHOFF, Eberhard. Ethik des Lebens. Grundlagen und neue Herausforderungen. Zweite, aktualisierte Auflage. Freiburg im Breisgau: Herder, 2013, p. 284-287. 
grossesse après l'IVF, avortement, banque d'embryons congelés, donation d'embryons «malades» pour la recherche et enfin eugénisme ${ }^{31}$.

En outre, étant donné qu'il n'est pas facile d'établir les limites entre l'amélioration de quelques membres de la société et la prévention médicale, les dilemmes de bioéthique posés par les PGD et PGS peuvent cependant être éclairés par deux catégories ontologiques de la bioéthique de la responsabilité jonassienne, lesquelles fonctionnent également aussi bien pour la mentalité séculière que pour la croyance religieuse juive ou chrétienne (cf. Gn 1, 2628), à savoir : l'image de l'être humain et la paternité en tant qu'archétype de la responsabilité. Les deux catégories fonctionnent pour la discussion en bio-éthique théologique ${ }^{32}$.

D'une part, l'image de l'être humain. Dans le livre Das Prinzip Verantwortung, Jonas remarque que «l'impératif de l'existence» est «l'impératif que soit une humanité ${ }^{33}$. Et pour que l'humanité soit vraiment dans l'avenir, il faut avoir maintenant une idée ontologique de l'être humain qui la soutient :

Avec ce premier impératif, nous n'avons pas à rendre des comptes à l'homme à venir, mais à l'idée de l'homme qui est telle qu'elle exige la présence des incarnations dans le monde. C'est en d'autres termes une idée ontologique qui ne garantit sans doute pas l'existence de son objet avec sa simple essence comme le fait soi-disant le concept de Dieu dans l'argument ontologique - loin de là ! - mais c'est une idée qui dit qu'une telle présence doit être, qui doit donc être protégée et qui nous en fait une obligation, à nous qui pouvons la mettre en péril. C'est cet impératif ontologique, résultant de l'idée de l'homme, qui sous-tend l'interdiction de jouer au va-tout avec l'humanité, une interdiction affirmée jusqu'alors sans avoir été fondée. Seule une idée de l'homme, en nous disant pourquoi des hommes doivent être, nous dit en même temps comment ils doivent être ${ }^{34}$.

${ }^{31}$ DABROCK, Peter. Bioethik des Menschen. In: HUBER, Wolfgang; MEIREIS, Torsten; REUTER, Hans-Richard (Hrsg.). Handbuch der Evangelischen Ethik. München: C.H. Beck, 2015, p. 549551.

32 SCHOCKENHOFF, 2013, p. 371.

33 JONAS, 2009, p. 94; JONAS, 2003, p. 90.

34 JONAS, 2009, p. 95, 250; JONAS, 2003, p. 91, 234.

Fronteiras, Recife, v. 4, n. 2, p. 533-553, jul./dez., 2021 
549 | La valeur ontologique de l'image et de la ressemblance de l'être humain avec Dieu...

L'être humain a un devoir ontologique envers « l'idée de l'homme » manifestée dans le monde. Il ne s'agit pas de maintenir l'essence de l'être humain, l'humanité, mais «l'idée ontologique » de l'être humain impose un devoir ontologique pour protéger l'existence humaine dans le monde comme une obligation, face aux périls que peut lui faire courir la technologie. L'idée ontologique de l'être humain fonctionne donc comme une idée directrice qui nous affirme le «pourquoi » et surtout le «comment» doit être l'être humain. Cependant, l'existence de l'être humain ne peut pas, après Kant, se soutenir par l'essence, comme dans le concept de Dieu de l'argument ontologique a priori de l'existence de Dieu, mais par l'existence ontologique $a$ posteriori de l'être humain dans le monde qui impose un devoir pour bien développer l'humanité.

D'autre part, Jonas souligne constamment dans le livre Das Prinzip Verantwortung que l'archétype de la responsabilité est la paternité ${ }^{35}$. D'après Jonas, la responsabilité parentale a un double sens complémentaire, le subjectif et l'objectif :

Souvenons-nous enfin que la sollicitude pour la progéniture est l'archétype humain élémentaire de la coïncidence entre la responsabilité objective et le sentiment de responsabilité subjectif, tellement spontanée qu'elle n'a pas besoin d'invoquer la loi morale, et que la nature nous a par avance éduqués en vue de types de responsabilité qui manquent de l'assurance de l'instinct et qu'elle y a préparé notre sentiment ${ }^{36}$.

Le soin de la progéniture constitue l'archétype humain de l'éthique parce qu'il concentre une responsabilité subjective (sentimentale) et une responsabilité objective (rationnelle) intrinsèques à la nature de l'être humain. Il ne s'agit ni de l'autonomie éthique donnée intérieurement par la raison ni de l'hétéronomie donnée extérieurement par la loi morale à l'être humain. C'est la nature elle-même qui nous a pourvus de la responsabilité grâce au sentiment et à la raison.

35 JONAS, 2009, p. 186, 200, 204, 209, 250, 257-259, 87-89 ; JONAS, 2003, p. 178, 189, $192-$ 193, 197, 234, 240-241, 84-86.

36 JONAS, 2009, p. 178; JONAS, 2003, p. 171. 
En ce qui concerne les techniques PGD et PGS, il faut rappeler aussi que Hans Jonas avait bien envisagé la problématique du diagnostic prénatal dans le cadre de l'eugénisme, dans l'article « Laßt uns einen Menschen klonieren : Von der Eugenik zur Gentechnologie » (1982), parce que le contrôle biologique de l'être humain, particulièrement le contrôle génétique, peut transformer la nature et l'image de l'être humain ; Jonas recommande donc «prudence» et « responsabilité »37. À cet égard, Jürgen Habermas souligne le pourquoi de la prudence et de la responsabilité : « Hans Jonas conclut, de sa présentation des choses, à l'irréversibilité et à l'autoréférentialité spécifiques de l'intervention dans un enchaînement complexe, autorégulé, aux conséquences largement incontrôlables ${ }^{38}$. C'està-dire qu'il il faut être prudent et responsable des conséquences de la mise en œuvre de la technoscience dans l'être humain.

\section{Considérations finales}

Finalement, l'image ontologique de l'être humain - forgée à partir du récit biblique de la création dans le livre de la Genèse (cf. Gn 1, 26-28) sollicite la protection responsable du statut ontologique de l'embryon comme un devoir pour que l'humanité soit, parce que dans la vulnérabilité de sa nature l'être humain peut manipuler génétiquement l'humanité dans l'avenir. Cependant, étant donné les opportunités qu'offrent les techniques PGD et la PGS pour éviter la souffrance et la douleur des enfants à cause des maladies et anormalités génétiques, la responsabilité est mise surtout aux mains des couples et parents afin d'éviter une série de risques qui peuvent aboutir socialement à l'eugénisme. En tant qu'idée directrice, l'idée ontologique de l'être humain permet de donner une orientation théologique et philosophique pour éclairer les dilemmes bioéthiques avec responsabilité, parce qu'elle nous affirme le «pourquoi » et le «comment» du devoir-être de l'existence humaine dans le monde, manifestée surtout dans la vulnérabilité de la vie

\footnotetext{
37 JONAS, 1985, p. 162.

38 HABERMAS, Jürgen. L'avenir de la nature humaine. Vers un eugénisme libéral ?. Paris: Gallimard, 2002, p. 74-75 ; HABERMAS, Jürgen. Die Zukunft der menschlichen Natur. Auf dem Weg zu einer liberalen Eugenik?. Frankfurt am Main: Suhrkamp, 2001, p. 84-85. 
d'un enfant. D'après le théologien Hans Küng, la question métaphysique posée par Jonas donne à penser l'éthique à partir de l'anthropologie :

Hans Jonas a raison de poser la question métaphysique à laquelle l'éthique n'avait jamais été confrontée jusqu'ici : pourquoi faudrait-il donc qu'il y ait une humanité, pourquoi devrait-on respecter son héritage génétique, pourquoi devraitil y avoir de la vie $?^{39}$.

La «bioéthique de la responsabilité selon Hans Jonas» montre son actualité pour la théologie en ce qui concerne la question ontologique fondamentale de la vie sur terre, la manipulation génétique et la préservation de l'image de l'être humain ${ }^{40}$. C'est aussi le noyau éthique de l'impératif catégorique-existentiel jonassien, lequel délie des prémisses religieuses ${ }^{41}$. Il s'agit enfin de préserver « la dignité de la personne, l'intégrité de l'image de l'homme » ou en termes religieux de l'« imago dei »: « die Würde der Person, Integrität des Menschenbildes religiös : der “imago dei" »42.

\section{Referências}

DABROCK, Peter. Bioethik des Menschen. In: HUBER, Wolfgang; MEIREIS, Torsten; REUTER, Hans-Richard (Hrsg.). Handbuch der Evangelischen Ethik. München: C.H. Beck, 2015, p. 537-583.

HABERMAS, Jürgen. Die Zukunft der menschlichen Natur. Auf dem Weg zu einer liberalen Eugenik?. Frankfurt am Main: Suhrkamp, 2001.

HABERMAS, Jürgen. Im Sog der Technokratie. Kleine Politische Schriften XII. Frankfurt am Main: Suhrkamp, 2013.

HABERMAS, Jürgen. L'avenir de la nature humaine. Vers un eugénisme

\footnotetext{
39 KÜNG, Hans. Projet d'éthique planétaire. La paix mondiale par la paix entre les religions. Paris : Seuil, 1991, p. 60, 92, 236; KÜNG, Hans. Projekt Weltethos. München: Piper, 1990, p. 52, 76, 185.

40 QUESADA-RODRÍGUEZ, Francisco. La bioética de la responsabilidad según Hans Jonas. Madrid: Universidad Pontificia Comillas, 2018, p. 129-132.

${ }^{41}$ KÖRNER, Ulrich H. J. Bioethik nichtmenschlicher Lebensformen. In: HUBER, Wolfgang; MEIREIS, Torsten; REUTER, Hans-Richard (Hrsg.). Handbuch der Evangelischen Ethik. München: C.H. Beck, 2015, p. 601.

42 JONAS, Hans. Technik, Medizin und Ethik. Zur Praxis des Prinzips Verantwortung. Frankfurt am Main: Suhrkamp,1985, p. 12.
} 
libéral ?. Paris: Gallimard, 2002.

JONAS, Hans. Das Prinzip Verantwortung. Versuch einer Ethik für die technologische Zivilisation. Frankfurt am Main : Suhrkamp, 2003.

JONAS, Hans. Ethics and Biogenetic Art. Social Research, v. 71, n. 3, p. 569$582,2004$.

JONAS, Hans. Heidegger and the Theology. In: JONAS, Hans. The Phenomenon of Life. Toward a Philosophical Biology. Illinois: Northwestern University Press, 1996, p. 235-261.

JONAS, Hans. La Religion Gnostique. Le message du Dieu étranger et les débuts du christianisme. Paris : Flammarion, 1978.

JONAS, Hans. Le principe responsabilité. Une éthique pour la civilisation technologique. Paris : Flammarion, 2009.

JONAS, Hans. Technik, Medizin und Ethik. Zur Praxis des Prinzips Verantwortung. Frankfurt am Main: Suhrkamp, 1985.

JONAS, Hans. The Concept of God after Auschwitz. In: JONAS, Hans. Mortality and Morality. A Search for the Good after Auschwitz. Illinois: Northwest University Press, 1996, p. 131-143.

JONAS, Hans. The Gnostic Religion. The Message of the Alien God and the Beginnings of Christianity. Boston: Beacon Press, 2001.

JONAS, Hans. Wie können wir unsere Pflicht gegen die Nachwelt und die Erde unabhängig vom Glauben begründen?. In: BÖHLER, Dietrich;BRUNE, Jens Peter. Orientierung und Verantwortung. Begegnungen und Auseinandersetzungen mit Hans Jonas. Würzburg: Königshausen \& Neumann, 2004, p. 71-84.

JONAS, Hans. Heidegger et la théologie. In : JONAS, Hans. Le phénomène de la vie. Vers une biologie philosophique. Bruxelles : De Boeck, 2001.

KÖRNER, Ulrich H. J. Bioethik nichtmenschlicher Lebensformen. In: HUBER, Wolfgang; MEIREIS, Torsten; REUTER, Hans-Richard (Hrsg.). Handbuch der Evangelischen Ethik. München: C.H. Beck, 2015, p. 585-647.

KÜNG, Hans. Projekt Weltethos. München: Piper, 1990.

KÜNG, Hans. Projet d'éthique planétaire. La paix mondiale par la paix entre les religions. Paris : Seuil, 1991. 
553 | La valeur ontologique de l'image et de la ressemblance de l'être humain avec Dieu...

LEHMANN, Karl. Auslotungen. Lebensgestaltung aus dem Glauben heute. Freiburg im Breisgau: Herder, 2016.

LEIBNIZ, Gottfried Wilhelm. Principes de la nature et de la grâce fondés en raison. Principes de la philosophie ou Monadologie. Paris : Presses Universitaires de France, 1954.

POLIWDA, Sebastian. Versorgung von Sein. Die philosophischen Grundlagen der Bioethik bei Hans Jonas. Zürich/New York: Georg Olms Verlag, 2005.

QUESADA-RODRÍGUEZ, Francisco. La bioética de la responsabilidad según Hans Jonas. Madrid: Universidad Pontificia Comillas, 2018.

RATH, Matthias Rath. La triple signification du mot "valeur" dans Das Prinzip Verantwortung de Hans Jonas et la Psychologisation en éthique. In : HOTTOIS, Gilbert ; PINSART, Marie-Geneviève (Éds.). Hans Jonas. Nature et responsabilité. Paris: Vrin, 1993.

SCHOCKENHOFF, Eberhard. Ethik des Lebens. Grundlagen und neue Herausforderungen. Zweite, aktualisierte Auflage. Freiburg im Breisgau: Herder, 2013.

Trabalho submetido em 01/08/2021. Aceito em 15/11/2021.

Francisco Quesada Rodriguez

Théologien et bioéthicien. Docteur en théologie de l'Université catholique de Louvain, Belgique. Master of Science en bioéthique à I'Université du Costa Rica; Master en sciences humaines à l'Université latine du Costa Rica. II a étudié la philosophie, la théologie spéculative et la bioéthique de Hans Jonas. II est l'auteur du livre La antropología filosófica de Hans Jonas. Ontología y ética de la responsabilidad (Madrid, Fundación Emmanuel Mounier, 2015) et La bioética de la responsabilidad según Hans Jonas (Madrid, Universidad Pontificia Comillas, 2018). E-mail: francis.quesada@gmail.com 